\title{
Estudo Comparativo da Eficiência de Diferentes Técnicas de Mensuração da Concentração Espermática em Suínos
}

\author{
Wagner Loesch Vianna ${ }^{2}$, Daniel Gonçalves Bruno ${ }^{1}$, André Namindome ${ }^{1}$, Aline Campos Rosseto ${ }^{2}$, \\ Paulo Henrique Mazza Rodrigues ${ }^{3}$, Marcos Eduardo Pinese ${ }^{2}$, Aníbal de Sant'Anna Moretti ${ }^{3}$
}

RESUMO - A inseminação artificial (IA) em suínos é uma técnica cada vez mais utilizada na suinocultura moderna, pois propicia ao suinocultor vantagens quanto ao desempenho dos reprodutores, ao controle dos cruzamentos e à facilidade na introdução de material genético. Objetivando o aperfeiçoamento da técnica de IA, foram avaliadas a acurácia, a precisão e a robustez do Espermiodensímetro (ESPERMIO) e do Espectrofotômetro (ESPECTRO), em comparação à Câmara de Neubauer. Utilizaram-se 142 ejaculados, que revelaram concentrações espermáticas médias (em milhões de sptz/mL) e número médio de doses que poderiam ser produzidas, respectivamente, de 229,1 e 22,6; 185,0 e 18,5; 283,6 e 28,0 para a Câmara de Neubauer, o Espectrofotômetro e o Espermiodensímetro. O viés médio (acurácia) e o desvio-padrão do viés médio (precisão) do ESPECTRO, em comparação à Câmara de Neubauer, foram, respectivamente, de - 44,1 e 52,6, enquanto para o ESPERMIO foram de 54,5 e 44,8 (milhões de sptz/mL). Pode-se inferir que o ESPECTRO tende a subestimar e o ESPERMIO, a superestimar a concentração espermática, em comparação à Câmara de Neubauer. O ESPECTRO foi mais acurado e igualmente preciso que o ESPERMIO. A influência do volume de ejaculado sobre os resultados do ESPERMIO e do ESPECTRO pode ser comprovada pelos resultados de robustez para o volume do ejaculado.

Palavras-chave: acurácia, câmara de neubauer, concentração espermática, espectrofotômetro, espermiodensímetro, precisão

\section{Efficiency of Different Measurement Techniques of Sperm Concentration in Swine}

\begin{abstract}
Artificial Insemination (AI) in swines is a rising technique that has been used during the last years at the swine production systems, because it furnish several advantages to the producers just like boar high efficiency, better breeding control and easiness in introducing foreign genetic materials. Accuracy, precision and robustness for ejaculation volume of spermdensimeter (ESPERMIO) and spectrofotometer (ESPECTRO) in relation to the Neubauer Count, designed as the standard technique, were evaluated objectifying higher performance of AI technique. A total of 142 boar ejaculation was used for spermatic concentration measurement by the techniques described above. The average spermatic concentrations $\left(10^{6} \mathrm{sptz} / \mathrm{mL}\right)$ and average doses number were, respectively: 229.1 and 22.6, 185.0 and 18.5, 283.6 and 28.0 for Neubauer count, Spectrofotometer and Spermdensimeter. ESPECTRO mean bias (accuracy) and bias standard deviation (precision), in relation to the Neubauer count were, respectively - 44.1 and 52.6, while ESPERMIO were 54.4 and 44.8. Through these results its possible to conclude that ESPECTRO has a tendency to subestimate and ESPERMIO to superestimate the spermatic concentration in relation to the Neubauer Count, and that the ESPECTRO was more accurate and less precise than ESPERMIO. Positive effect of ejaculation volume on accuracy and precision in both techniques (ESPERMIO and ESPECTRO) was confirmed by the robustness results.
\end{abstract}

Key Words: accuracy, neubauer count, precision, spectrofotometer, sperm concentration, spermdensimeter

\section{Introdução}

A inseminação artificial (IA) - uma biotécnica utilizada na reprodução da espécie suína desde a década de 30 - tem evoluído progressivamente, demonstrando sua influência na eficiência reprodutiva dos sistemas de produção. Assim como a IA é de vital importância para a suinocultura atual, a determinação da qualidade do sêmen utilizado é essencial, pois, problemas de infertilidade e subfertilidade refletem no aumento de retorno do cio, taxa de parto e tamanho da leitegada diminuídos (García Ruvalcaba et al., 1999).

Para a eficiência de um programa de IA, um fator que deve ser considerado é a concentração do sêmen do macho destinado à reprodução, cuja estimativa deve ser precisamente determinada. A superestimação

\footnotetext{
${ }^{1}$ Bolsista de Iniciação Científica da FAPESP e aluno da graduação da FMVZ - USP

2 Pós-graduando do Departamento de Reprodução Animal da FMVZ - USP. E.mail: waloesch@fmvz.usp.br

${ }^{3}$ Professor Livre-Docente do Departamento de Nutrição e Produção Animal da FMVZ - USP. E.mail: adsamore@usp.br
} 
significa que a concentração mensurada é maior que a concentração real do ejaculado, o que propicia número menor que o ideal de espermatozóides que conseguem atingir o oviduto e estar presentes no momento da ovulação, uma vez que o útero impõe entraves à passagem desses espermatozóides caracterizada pelo refluxo, pela fagocitose por células de defesa e por perdas durante o percurso (Krueger et al., 1999). Outra conseqüência da superestimação é que poderá ser adicionando mais diluidor e, com isso, provocar diminuição na quantidade de plasma seminal da dose, pois estes influem no pico de LH na fêmea e, conseqüentemente, na maturação oocitária final e no momento da ovulação (Stratman \& Self, 1960; Weitze et al., 1990). Esses fatores, em conjunto ou isoladamente, podem levar à queda da fertilidade no rebanho, pelo incremento do retorno ao estro, por falhas na fertilização, ou ainda pela diminuição da taxa de parto e no tamanho da leitegada, acarretando, portanto, prejuízos ao criador.

Caso a concentração espermática seja subestimada, ou seja, se a concentração mensurada for menor que a concentração real, haverá, da mesma forma, prejuízos na granja e, por conseqüência, aumento do custo da dose. Além disso, falhas de manejo reprodutivo dos machos podem ser mascaradas, se houver subestimação da concentração espermática, já que as concentrações mais altas que 2 a 3,5 × $10^{9}$ espermatozóides preconizadas podem aumentar os índices de fertilidade.

Neste estudo, elegeu-se a Câmara de Neubauer como a técnica padrão, porque proporciona avaliação precisa, exigindo, no entanto, maior demanda no tempo de análise (Brillard \& McDaniel, 1985; Paulenz et al., 1995). Essa técnica é recomendada pela "World Health Organization" (WHO) (Paulenz et al., 1995; Shannon \& Vishwanath, 1995; Steverink et al., 1998). Todavia, segundo Evenson et al. (1993), essa precisão não é tão exata, observando-se diferenças de até $20 \%$ entre a contagem duplicada de uma mesma amostra, feita por um mesmo técnico. Por outro lado, Paulenz et al. (1995) apontam coeficiente de variação de $12,3 \%$ e Sokol et al. (2000), de apenas $10 \%$.

Outra vantagem desse método é o baixo custo (Tomlinson et al., 2001), além de ser o único método direto que permite a contagem da célula espermática (Evenson et al., 1993).

Além do tempo consumido, existem outras desvantagens inerentes a essa técnica que associam menor precisão ao aumento da diluição, pois há sobreposição e desuniformidade ao se preencher a câmara. A distribuição dos espermatozóides pela câmara é, portanto, importante e deve ser heterogênea (Paulenz et al., 1995). Dessa forma, mesmo considerando a Câmara de Neubauer a técnica padrão, devem-se respeitar as vantagens e desvantagens de sua utilização.

O espermiodensímetro, na realidade, oferece estimativa muito rápida da concentração espermática, com base no grau de turbidez ou densidade do ejaculado. A literatura não menciona dados que indiquem a precisão desse método, nem das vantagens ou desvantagens. Em razão da carência de informações e da necessidade de se avaliarem o grau de precisão e a relação direta com a eficiência espermática nas criações comerciais, despertou-se o interesse na averiguação.

As hipóteses levantadas apontam a menor precisão do espermiodensímetro comparado com os outros dois métodos, pois a leitura apresenta maior subjetividade, além da própria densidade do ejaculado ser influenciada pelo número de leucócitos, sujidades (oriundas do próprio equipamento ou da coleta de sêmen) e células de descamação.

O espectrofotômetro consiste de uma técnica em que a leitura da concentração é feita através do grau de absorbância da luz no ejaculado. Trata-se de um método mais rápido que o da Câmara de Neubauer, com a desvantagem de que as concentrações de espermatozóides só podem ser avaliadas dentro de certa faixa. Além disso, a curva de calibração não é válida para todos os cachaços, em razão das diferenças individuais, pois a quantidade de luz difundida pelos espermatozóides em suspensão depende de seu tamanho, forma e índice de refração. Assim como no espermiodensímetro, o espectrofotômetro sofre influência de células de descamação, leucócitos e sujidades.

A relação linear entre a densidade óptica e a espermática oferece um método rápido para estimativa do número de espermatozóides, que, no entanto, perde parte de sua precisão com o sêmen de mamíferos, em razão da presença de proteínas plasmáticas (Ciereszko \& Dabrowski, 1993).

O coeficiente de variação do espectrofotômetro é muito baixo em comparação com o da Câmara de Neubauer, tornando a técnica menos subjetiva (Paulenz et al., 1995).

Com base nessas informações, é muito importante que se tenha à disposição meios eficientes e precisos 
para quantificar o número de espermatozóides do ejaculado de cachaços destinados à reprodução. Objetivou-se, com este trabalho, estudar a acurácia, a precisão e a robustez, no volume do ejaculado, a partir da concentração espermática obtida pelas técnicas do Espermiodensímetro (ESPERMIO) e do Espectrofotômetro (ESPECTRO), em comparação à Câmara de Neubauer (CN).

\section{Material e Métodos}

O experimento foi realizado em uma Central de Inseminação Artificial de um Sistema de Produção de Suínos, com 10.000 matrizes, localizada no município de Cerqueira César, SP. Selecionaram-se, pelos exames andrológicos, 55 machos reprodutores suínos de diferentes linhagens, alojados em baias individuais. Foram coletados 142 ejaculados pela técnica da "mão enluvada", utilizando-se um copo térmico $\left(36^{\circ} \mathrm{C}\right)$ para acondionamento de um Becker graduado. Em todo ejaculado coletado, foram mensurados volume e concentração espermática pelas seguintes técnicas: Câmara de Neubauer, Espermiodensímetro (Minitüb Inc.) e Espectrofotômetro (Micronal B340 "520 nm").

A técnica da Câmara de Neubauer foi definida como padrão, sendo a acurácia das técnicas alternativas (ESPECTRO e ESPERMIO) calculada pela média do viés, obtida pela diferença entre a concentração da técnica alternativa e a concentração da técnica padrão. Observa-se que, quanto mais próximo de zero é o viés, mais acurado é o teste em relação à CN. A precisão de cada técnica em relação à $\mathrm{CN}$ foi determinada pelo desvio-padrão dos vieses entre a técnica alternativa e a técnica padrão (quanto menor o desvio-padrão mais preciso é o teste). Testou-se a robustez das técnicas ESPECTRO e
ESPERMIO, em função do volume de cada ejaculado, onde foi realizada uma análise de regressão simples da variável dependente, viés do ESPECTO ou ESPERMIO, em relação à técnica padrão, em função do volume do ejaculado. Para comparar a precisão de ESPECTRO e ESPERMIO, utilizou-se o teste de Bartlett de homogeneidade das variâncias. As acurácias entre as técnicas alternativas foram comparadas empregando-se a análise de variância com os dados dos vieses entre a técnica alternativa e a padrão. Adicionalmente, o teste T, para média igual a zero, foi utilizado para o viés médio de cada técnica com o valor zero. Neste caso, resultado significativo indica falta de acurácia da técnica.

\section{Resultados e Discussão}

No total de 142 ejaculados analisados, os resultados descritivos das técnicas encontram-se na Tabela 1. O espectrofotômetro apresentou valores inferiores aos verificados na Câmara de Neubauer, havendo, portanto, subestimação da concentração espermática, quando comparada à técnica padrão, ao passo que o espermiodensímetro revelou valores superiores aos da Câmara de Neubauer, com tendência à superestimativa da concentração espermática.

Os resultados de viés médio, indicadores de acurácia, e desvio-padrão do viés, indicadores de precisão, para o espermiodensímetro e espectofotômetro são descritos na Tabela 2. Notou-se, novamente, habilidade deoespectrofotômetro subestimareo espermiodensímetro, superestimar a concentração espermática, em comparação à Câmara de Neubauer. No entanto, o viés médio do espectrofotômetro foi mais próximo de zero, ou seja, em média, o espectrofotômetro tendeu a ser mais acurado que o espermiodensímetro. Entre-

Tabela 1 - Resultados descritivos das técnicas de mensuração da concentração espermática

Table 1 - Descriptive results of spermatic concentration measurement techniques

\begin{tabular}{|c|c|c|c|c|}
\hline $\begin{array}{l}\text { Técnica } \\
\text { Technique }\end{array}$ & $\mathrm{N}$ & $\begin{array}{l}\text { Média concentração }\left(10^{6} \mathrm{sptz} / \mathrm{mL}\right) \\
\text { Average concentration }\left(10^{6} \mathrm{sptz} / \mathrm{mL}\right)\end{array}$ & $\begin{array}{l}\text { Desvio-padrão } \\
\text { Standard deviation }\end{array}$ & $\begin{array}{l}\text { Número médio de doses }{ }^{1} \\
\text { Average number of doses }{ }^{1}\end{array}$ \\
\hline $\begin{array}{l}\text { Câmara de Neubauer } \\
\text { Neubauer Count }\end{array}$ & 142 & 229,1 & 102,5 & 22,6 \\
\hline $\begin{array}{l}\text { Espectrofotômetro } \\
\text { Spectrofotometer }\end{array}$ & 142 & 185,0 & 68,2 & 18,5 \\
\hline $\begin{array}{l}\text { Espermiodensímetro } \\
\text { Spermdensimeter }\end{array}$ & 142 & 283,6 & 106,0 & 28,0 \\
\hline
\end{tabular}

1 Concentração espermática x volume/3000 (sem considerar a motilidade e dose fixa em 3 bilhões de sptz).

${ }^{1}$ Spermatic concentration $x$ volume/3000 (not considering the motility and fixed dose at 3 billion sptz).

R. Bras. Zootec., v.33, n.6, p.2054-2059, 2004 (Supl. 2) 
tanto, com relação à precisão, não foi possível mostrar diferença no desvio-padrão do viés, por intermédio do teste de homogeneidade das variâncias, indicando precisão semelhante entre as técnicas. Como descrito anteriormente, o ESPERMIO obteve concentração maior que a técnica padrão, provavelmente pela influência de sujidades, número de leucócitos e células de descamação, que aumentariam a turbidez do sêmen. Com relação à tendência de o ESPECTRO obter valores de concentração menores que a técnica padrão, a curva de calibração do aparelho deve influir de maneira significativa na subestimação do resultado, visto que sofre as mesmas influências do ESPERMIO (sujidades, células de descamação e número de leucócitos), mas com menor grau de subjetividade. Recomenda-se, nos trabalhos rotineiros realizados a campo, sempre calibrar o aparelho de espectrofotômetro, em períodos fixos de tempo (por exemplo, a cada seis meses), por intermédio da câmara de Neubauer, com exames do ejaculado da própria granja.

Quanto ao efeito do número elevado de espermatozóides e volume da dose inseminante sobre a sobrevivência embrionária, Stratman \& Self (1960) observaram que, tanto aos três dias quanto aos 25 dias de inseminação, o número de espermatozóides (2,5; 5,0 e 10,0 x $10^{9}$ espermatozóides, diluídos em três doses de plasma seminal: 10, 20 e $50 \mathrm{~mL}$ ) não influenciou o número de embriões, em decorrência das altas concentrações utilizadas.

Tabela 2 - Acurácia e precisão do espermiodensímetro e espectrofotômetro

Table 2 - Accuracy and precision of spermdensimeter and spectrofotometer

\begin{tabular}{|c|c|c|}
\hline $\begin{array}{l}\text { Técnica } \\
\text { Technique }\end{array}$ & $\begin{array}{c}\text { Viés médio }{ }^{1} \\
\text { Average of bias }\end{array}$ & $\begin{array}{l}\text { Desvio-padrão } \\
\text { do viés } \\
\text { Standard deviation } \\
\text { of bias }\end{array}$ \\
\hline $\begin{array}{l}\text { Espectrofotômetro } \\
\text { Spectrofotometer }\end{array}$ & $-44,1 b$ & $52,6 a$ \\
\hline $\begin{array}{l}\text { Espermiodensímetro } \\
\text { Spermdensimeter }\end{array}$ & 54,5a & $44,8 \mathrm{a}$ \\
\hline \multicolumn{3}{|c|}{$\begin{array}{l}\text { Colunas com distintas letras sobrescritas diferem entre si } \\
(P<0,0001) \text {. } \\
1 \text { Diferem estatisticamente de zero }(P<0,0001) \text {. } \\
\text { Different superscripts within the column are different }(P<.0001) \text {. } \\
{ }^{1} \text { Different from zero }(P<.0001) \text {. }\end{array}$} \\
\hline
\end{tabular}

R. Bras. Zootec., v.33, n.6, p.2054-2059, 2004 (Supl. 2)
Para que a fertilização seja efetiva, um número suficiente de espermatozóides deve estar presente no reservatório de sêmen que se forma na região caudal do istmo. Os espermatozóides devem percorrer em torno de 1 a 2 m pelos cornos uterinos no fluido da dose inseminante, auxiliados principalmente pela contração longitudinal do útero, até alcançarem o local da fertilização. No entanto, há no útero uma série de fatores que interferem negativamente, como refluxo, fagocitose pelos leucócitos presentes no útero, contração uterina e perda de espermatozóides resultante do rápido transporte na cavidade abdominal (Krueger et al., 1999). First et al. (1968) observaram que, decorridos 15 minutos da inseminação, menos da metade dos espermatozóides foi recuperada no útero.

Alguns desses fatores que impedem a chegada dos espermatozóides ao local de fertilização são, no entanto, controversos. Há evidências de que a fagocitose só ocorre após a morte dos espermatozóides por outros processos, pois esta se inicia-se 8 horas após a inseminação, enquanto a perda de motilidade da maioria dos espermatozóides, entre 2 e 4 horas após (First et al., 1968). Outros estudos demonstraram que a fagocitose ocorre 2 horas após a inseminação (Steverink et al., 1998). Viring \& Einarsson (1981) destacaram que pode haver perdas decorrentes da rápida passagem de sêmen para a cavidade abdominal.

Outra barreira contra a passagem de espermatozóides para o local de fertilização é o refluxo, na medida em que menos células ficam disponíveis. A variação do volume do refluxo atinge de 1 a 56\% no momento da inseminação; de 3 a 16\% 30 minutos após a inseminação; e de 1 a $94 \%$ de 30 minutos a 2 horas. A concentração média de espermatozóides em relação à dose inseminante, no refluxo, atinge valores respectivos de $65 \pm 2,6 \%$, $40 \pm 1,5 \%$ e $26 \pm 1,4 \%$, em razão de a correlação entre o volume e o número de espermatozóides ser alta $(r=0,97, r=0,73$ e r=0,87). Conclui-se que o volume do refluxo é indicativo do número de espermatozóides no refluxo e influi negativamente na porcentagem de embriões normais $(\mathrm{P}<0,05)$, quando dosagem subótima ( $10^{9}$ espermatozóides) é usada, o que ressalta a importância de se medir precisamente a concentração de sêmen para não se ter prejuízos decorrentes da inseminação (Steverink et al., 1998).

O fato de a concentração do refluxo ser alta e próxima do momento da inseminação foi evidenciado em experimento com porcas em sistema de monta 
natural, havendo perda de até $1 / 3$ dos espermatozóides do ejaculado em 2 horas, pelo refluxo (Paulenz et al., 1995).

Existem evidências de que o tamanho do útero influi no volume do refluxo, posto que porcas que pariram uma vez tiveram refluxo maior $(>20 \mathrm{~mL}$ ) que aquelas que pariram mais vezes (Steverink et al., 1998).

Em razão desses fatores impedirem que grande parte dos espermatozóides chegue ao local de fertilização, infere-se que a conseqüência do uso de doses menores que 2 a $3,5 \times 10^{9}$ espermatozóides pode implicar em diminuição das taxas de fertilidade das fêmeas. Inseminações com $5 \times 10^{9}$ e $10 \times 10^{9}$ espermatozóides resultaram em maior número de espermatozóides no oviduto e na maior porcentagem de oócitos fertilizados que inseminações com $1 \times 10^{9}$ espermatozóides/dose (Baker et al., 1968). Da mesma forma, inseminações com dose menor de sêmen $\left(10^{9} \mathrm{sptz} /\right.$ dose $)$ apresentaram porcentagem mais alta de animais com resultados moderados na fertilização (90 a 80\% de embriões normais), quando o intervalo da inseminação à ovulação foi de 24 a 36 horas. No intervalo de 12 a 24 horas, a dose de $1 \times 10^{9}$ resultou em $26 \%$ menos porcas com resultados satisfatórios na fertilização (100-90\% de embriões normais) e 19\% mais porcas com resultados moderados de fertilização, quando comparado com uma dose de $3 \times 10^{9}$ espermatozóides. Em geral, a dose tende a afetar a

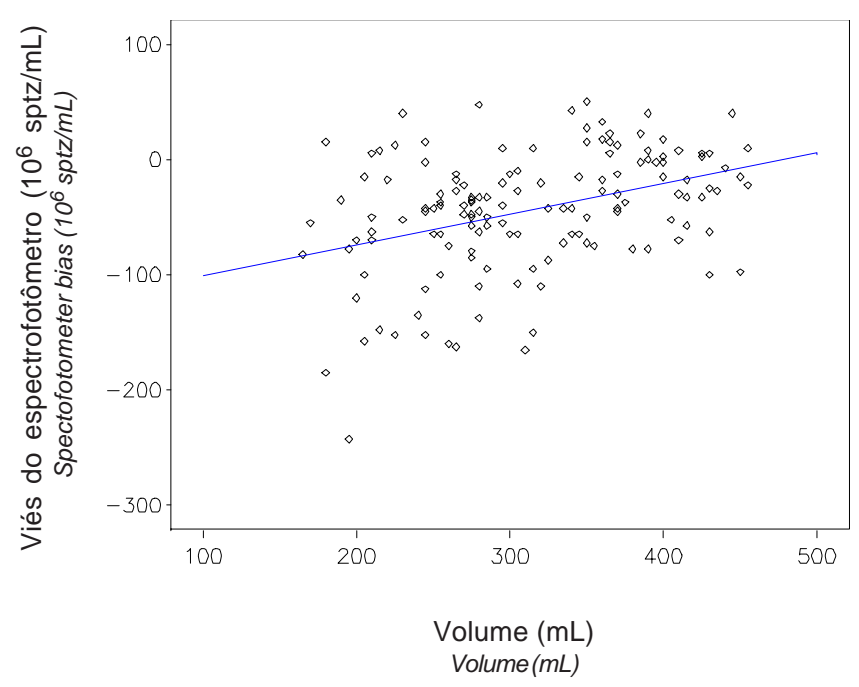

Figura 1 - Robustez do espectrofotômetro. Figure 1 - Robustness of spectrofotometer.

R. Bras. Zootec., v.33, n.6, p.2054-2059, 2004 (Supl. 2) porcentagem de embriões normais, sendo, respectivamente, de $78 \pm 0,3 \%$, $84 \pm 0,3 \%$ e $91 \pm 0,4 \%$ para doses iguais a $1 \times 10^{9}, 3 \times 10^{9}$ e $6 \times 10^{9}$ (Steverink et al., 1997).

Em estudo que relacionava parâmetros que prediziam a qualidade do sêmen dos cachaços com a fertilização in vivo, utilizando-se doses ótimas e sub-ótimas de sêmen (respectivamente, $3 \times 10^{9}$ e $0,3 \times 10^{9}$, sendo a última obtida mediante diluição 10 vezes maior que a dose de $3 \times 10^{9}$ em "Beltsville Tawing Solution"), verificou-se que a taxa de ovulação era menor com a dose de $0,3 \times 10^{9}$, conforme mostrado pelo número de corpos lúteos $(12,2 \pm 3,5$ e $8,6 \pm 4,2$, respectivamente, para as doses ótimas e subótimas). Esse resultado foi atribuído, possivelmente, à diminuição dos componentes do plasma seminal com o aumento da diluição (Tardiff et al., 1999).

A robustez para volume do ejaculado, avaliada por meio do espectrofotômetro e do espermiodensímetro, consta nas Figuras 1 e 2, respectivamente.

Pode-se verificar, nas Figuras 1 e 2, que o volume do ejaculado exerceu pequeno efeito sobre o viés de ambos ESPECTRO e ESPERMIO. Portanto, infere-se que, quanto maior o volume do ejaculado, maior será a acurácia dos testes, em relação à Câmara de Neubauer, em decorrência, provavelmente, da diluição dos efeitos de sujidades, células de descamação e número de leucócitos com o aumento de volume do ejaculado.

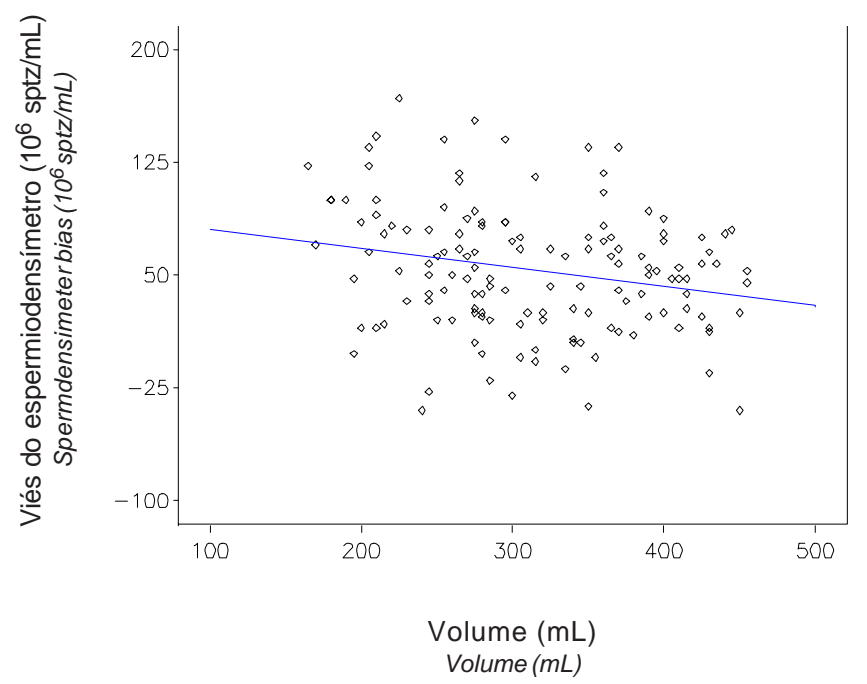

Figura 2 - Robustez do espermiodensímetro. Figure 2 - Robustness of spermdensimeter. 


\section{Conclusões}

O espectrofotômetro tende a subestimar e o espermiodensímetro, a superestimar a concentração espermática em comparação à Câmara de Neubauer. Assim, ao utilizar o ESPECTRO, o potencial dos reprodutores de um sistema de produção seria subutilizado e, com o ESPERMIO, seriam produzidas doses menores que o padrão, o que pode originar problemas de fertilização.

Quanto maior o volume do ejaculado, maior será a acurácia de ambos ESPECTRO e ESPERMIO, em comparação à Câmara de Neubauer.

\section{Agradecimento}

À Fundação de Amparo à Pesquisa do Estado de São Paulo, pelo financiamento da pesquisa, e à Granja Rosseto, pela disposição de sua infra-estrutura e animais.

\section{Literatura Citada}

BAKER, R.D.; DZIUK, P.J.; NORTON, H.W. Effect of the volume of semen and drugs on transport of sperm in artificial inseminated gilts. Journal of Animal Science, v.27, p.88-93, 1968.

BRILLARD, J.P.; MCDANIEL, G.R. The realiability and efficience of various methods for estimating spermatozoa concentration. Poultry Science, v.64, p.155-158, 1985.

CIERESZKO, A.; DABROWSKI, K. Estimation of sperm concentration of rainbow trout, whitefish and yellow perch using a spectrophotometric technique. Aquaculture, v.109, p.367-373, 1993.

EVENSON, D.P.; PARKS, J.E.; KAPROTH, M.T. et al. Rapid determination on sperm cell concentration in bovine semen by flow cytometry. Journal of Dairy Science, v.76, p.86-94, 1993.

FIRST, N.L.; SHORT, R.E.; PETER, J.B. et al. Transport and loss of boar spermatozoa in the reproductive tract of the sow. Journal of Animal Science, v.27, p.1037-1040, 1968.

GARCÍA RUVALCABA, J.A.; LAPUENTE, S.; CORCUERA, D. et al. Avaliação prática do sêmen. Suinocultura Industrial, n.21, p.32-35, 1999.

KRUEGER, C.; RATH, D.; JOHNSON, L.A. Low dose insemination in synchronized gilts. Theriogenology, v.52, p.1363-1373, 1999.
PAULENZ, H.; GREVLE, I.S.; TVERDAL, A. et al. Precision of the Coulter Counter for routine assessment of boar-sperm concentration in comparison with the haemocytometer and spectrophotometer. Reproduction in Domestic Animals, v.30, p.107-111, 1995.

SHANNON, P.; VISHWANATH, R. The effect of optimal and suboptimal concentrations of sperm on the fertility of fresh and frozen bovine semen and a theoretical model to explain the fertility differences. Animal Reproduction Science, v.39, p.1-10, 1995.

SOKOL, R.Z.; SHULMAN, P.; PAULSON, R.J. Comparison of two methods for the measurement of sperm concentration. Fertility and Sterility, v.73, p.591-594, 2000.

STEVERINK, D.W.B.; SOEDE, N.M.; BOUWMAN, E.G. et al. Influence of insemination-ovulation interval and sperm cell dose on fertilization in sows. Journal of Reproduction and Fertility, v.111, p.165-171, 1997.

STEVERINK, D.W.B.; SOEDE, N.M.; BOUWMAN, E.G. et al. Semen backflow after insemination and its effect on fertilization results in sows. Animal Reproduction Science, v.54, p.109119, 1998.

STRATMAN, F.W.; SELF, H.L. Effect of semen volume and number of sperm on fertility and embryo survival in artificially inseminated gilts. Journal of Animal Science, v.19, p.10811088, 1960.

TARDIFF, S.; LAFOREST, J.P.; CORMIER, N. et al. The importance of porcine sperm parameters on fertility in vivo. Theriogenology, v.52, p.447-459, 1999.

TOMLINSON, M.; TURNER, J.; POWELL, G. et al. One-step disposable chambers for sperm concentration and motility assessment: how do they compare with the World Health Organization's recommended methods?. Human Reproduction, v.16, p.121-124, 2001.

VIRING, S.; EINARSSON, S. Sperm distribution within the genital tract of naturally inseminated gilts. Nord. Vet. Med., v.33, p.145-149, 1981.

WEITZE, K.F.; LOTZ, J.H.; EVERWAND, A. et al. Interaction between inseminate, uterine and ovarial function in the sow. II. Investigations of ovulation by the use of sperm-free media. Reproduction in Domestic Animals, v.25, p.197-204, 1990.

Recebido em: 03/06/03 Aceito em: 29/04/04 